\section{PROPHYLACTIC USE OF VACCINES IN THE GREAT WAR *}

\author{
N. E. WAYSON, M.D. \\ Assistant Surgeon, United States Public Health Service \\ WASHINGTON, D. C.
}

The diseases which are perhaps most detrimental to the efficiency of military bodies are those of the intestinal tract. To the ubiquitous dysentery and typhoid fever are added in Europe the paratyphoid fevers and cholera. It is particularly to combat these diseases that boiling of the drinking water, chlorination of the water supply, disinfection of the dejecta, and separation of the infected with or without symptoms, together with earlier and accurate bacteriologic diagnosis, have been so intensively and thoroughly practiced in the military bodies now fighting. With the manifold exigencies of war, however, these precautions are not sufficient, and the individual fighting unit must be given detailed attention. The failure of these general sanitary measures during this war involving trench fighting, together with previous military experience with antityphoid vaccination, was the incentive for attempts at immunization of the individual. Hence, in March, 1914, France adopted Labbé's legislation for compulsory vaccination against typhoid, and the execution of the law was vigorously prosecuted in October, 1914. The Russian Medical Association proposed similar vaccination in their army early in 1915, and the medical as well as some military authorities of England advocated compulsory antityphoid vaccination in the armies of Great Britain, at the beginning of the war. Reports show that the troops on the west front of the German army were vaccinated against typhoid in the late fall and winter of 1914 and 1915.

Anticholera vaccination has been practiced among those troops which were exposed in endemic areas, such as exist on the eastern front of the war zone.

Antidysentery vaccination has apparently not been widely practiced in the armies of the belligerents.

The problems involved in the attempts to immunize soldiers against these scourges have been summarized and emphasized by Theobald Smith. ${ }^{1}$ Some facts to which he gives prominence are:

The object of immunization is to raise the resistance of the body. . . No kind of vaccination is invariably and completely protective. We may, however, hope to convert a fatal into a nonfatal attack, a mild attack into one so mild as to remain unrecognized, or into complete protection.

The essential factor to be kept in mind is to raise such resistance with the least injury to the subject.

There is a clearly marked distinction between groups of organisms as to the capacity of the hosts to be immunized experimentally.

The object of immunization is to suppress the invader; the problem underlying is to find the precise aggressive weapons which prevent the invader from multiplying.

The principals of the group of organisms which concern us are those to which the hosts lend themselves well to experimental immunization; and one of the precise aggressive weapons is that which gives rise to the immune substances in the host, namely, the antigen.

* Read before the Section on Pharmacology and Therapeutics at the Sixty. Eighth Annual Session of the American Medical Association, New York, June, 1917.

1. Smith, Theobald: Underlying Problems of Immunizdtion Address bcfore the Congress of American Physicians and Surgeons, 1916.

\section{ANTIGEN}

The antigens or vaccines used in the various armies and military bodies have been as numerous as are the peoples at war. The preparation of these vaccines, however, has had for its guiding principle the conservation of all the "immunogenic qualities" of the organisms involved, and the destruction of their infectivity. The latter has necessitated the use of dead or greatly attenuated bacteria.

The attenuation or killing has been variously accomplished by heating, by the application of chemicals (chiefly phenols and ether), by autolyzing, and by sensitizing with an immune serum. Conservation of the "immunogenic" substances (some thermolabile) has been accomplished by using that degree of heat which closely approximates the thermal death point of the organism, erring on the side of too low a degree, and adding some chemical disinfectant as a preservative; by the use of chemicals alone; by autolysis, or by sensitization.

The English have made use of Wright's typhoid vaccine as modified by Leishman. This vaccine is monovalent (containing the one strain of the typhoid bacillus, the Rawling strain). The choice of this strain was dependent apparently only on its adaptability to homogeneous emulsification. The cultures are grown in plain broth for forty-eight hours, heated to $53 \mathrm{C}$., and made up to contain a 0.5 per cent dilution of compound solution of cresol. The vaccine contains a billion organisms to the cubic centimeter. Later the cultures were grown on agar and emulsified in salt solution. ${ }^{2}$

The Canadian contingents, at least some of them, were originally inoculated with vaccines made from agar cultures.

The French have used several vaccines, principally those of Chantemesse, Vincent and Besredka. Vincent states that the laboratory at Val-de-Grâce has supplied his vaccine to the armies of Greece, Italy, Serbia, Spain and Turkey. Vincent's vaccine is polyvalent (containing ten strains of the typhoid bacillus). The cultures are autolyzed in salt solution, and killed by exposure to ether for from thirty-five to forty minutes, which procedure extracts simultaneously many of the lipoidal substances contained in the organism. Vincent considers these lipoidal substances febrigenic and responsible in part for the local reactions.

The vaccine of Chantemesse is monovalent, made with agar cultures of from eighteen to twenty hours' incubation, emulsified in salt solution, and heated to 56 C. Tricresol is added to make a final dilution of 0.25 per cent. The vaccine contains a billion organisms per cubic centimeter.

Besredka's method is founded on the employment of a living, sensitized vaccine. The typhoid bacillus is grown for forty-eight hours on agar, emulsified, and antityphoid serum added. After twenty-four hours' exposure to the serum, the living organisms are decanted and washed, and then emulsified anew in physiologic sodium chlorid solution. The use of Besredka's vaccine seems to be limited.

Goldscheider states that Marx's vaccine was used in immunizing one of the divisions of the German army on the west front. This vaccine is apparently a heatkilled culture, emulsified in salt solution, to which phenol is added to make a final dilution of 0.5 per cent. The official German vaccine is made from agar cul-

2. Personal communication to the author. 
tures, suspended in salt solution, killed at $55 \mathrm{C}$., and sufficient phenol added to make 0.5 per cent. Each cubic centimeter contains one third of an oese.

The Russians originally employed Wright's antityphoid prophylactic, but later used a polyvalent vaccine.

The choice of strains is dependent on several factors. Some authors elect the use of a strain from the local epidemic, but all are in accord that strains should be chosen with regard to their antigenic value. Toxicity is considered desirable by some and undesirable by others. This disparity of opinion is based on the question of the relation which toxicity has to "immunogenic" qualities. Estimates of these qualities are at present based on tests of agglutinogenic and protective power in experimental animals.

The experience of the English and Canadians, and thus far of the Americans, shows no preferential efficacy of a polyvalent, as opposed to a monovalent typhoid vaccine.

Dosage.-The dosage of these vaccines varies but slightly in the total amount given, and in the intervals after which the individual doses are administered. The total amount inoculated ranges from 1,600 million to 4,000 million bacteria, in the practice generally in vogue, and the intervals of dosage vary from five to eight days. While this is stated as the practice, it must be recalled that many of these vaccinations are most irregular in the periodicity of dosage, and incomplete in the number of doses, and consequently in the total amount administered. This irregularity and incompleteness of the vaccination occurs through military exigency, fabrication and illness on the part of the subjects, and, to some extent, on a lack of appreciation of the necessity for thoroughness by the sanitary officers.

The number of doses varies from two to four or more. It is the opinion of several authors who have made analytic studies of the effects of the vaccination that three at most, and frequently two inocculations are as effective as four or more.

There seems, however, to be a growing tendency to increase the total amount of antigen, in the belief, as Weber concludes from his findings in experimental work, that "the mass of vaccine injected is important, not only from the point of view of the solidity of the immunity, but from the precocity of its appearance; the more the dose is increased the more sure is the animal inoculated to have an immunity which will resist infection."

Fiessinger, who writes from an apparently large experience, concludes his studies with the admonition, "Do not vaccinate lightly."

Widal and Salimbéni recommend large doses, even as much as 10 billion organisms at a single dose, and conclude from their expermental observations on man that the reaction is not more severe than with lighter dosage. Their advocacy of a single injection of a large amount is with the obvious appreciation of the necessary irregularity and incompleteness of a vaccination divided into several administrations, over periods which permit troops being moved great distances from the place of initial inoculation.

Reinoculation.-The period after which reinoculation is practiced has been dependent largely on Wright's observations in India, and has varied usually from two to four years. Experience has apparently been contrary to inoculation at such long intervals, and the present practice seems to be a revaccination at from eight months to a year. No reports of a second revaccination were found, although there are reports of individuals who gave an anamnesis of typhoid at 16 years of age, in their early twenties, and again in the army in their early thirties.

\section{VACCINATION}

Methods of Vaccination.-These are chiefly parenteral, though Lumière and Chevrotier have advocated, and used enterally, gelatin-coated pills of a dried mixed polyvalent typhoid-colon vaccine. This method has not yet had an extensive use, but the English commission has reported unfavorably concerning its efficiency. The advantages of such a method of administration are self-evident, provided it were effective.

The technic of the parenteral administration is that of hypodermic injection, practiced aseptically. The sites most frequently chosen are the subclavicular, deltoid and subspinous areas. The skin is painted with tincture of iodin, or washed with alcohol, 60 per cent., and injection is made subcutaneously. With a battery of a few syringes and several needles, which can be intermittently boiled, several hundred men can be inoculated within a short space of time. Rubbercapped phials or bottles are used as containers for the vaccine. The Canadians, having exhausted their supply of rubber caps, used finger cots rolled up to make a strong collar for the neck of the bottle, and paraffined these for storage and shipment. Hermetically sealed ampules are also used as containers of the vaccine.

Results of the Vaccination.-The results of these various vaccinations may be analyzed from the standpoint of the immunization problems emphasized by Smith. In other words, what are the immediate effects on the host, and what are the effects on the course of the disease, and on its dissemination, since there is no absolute immunity?

Reaction of the Host.-The effects of vaccination vary, from those of local reddening and edema, persisting for twenty-four hours and progressively disappearing, to a severe general reaction, marked by shock and collapse. The percentage of general reactions is perhaps well illustrated by the observations of Maurange on 39,215 injections; he found the results given in Table 1. As will be noted, he does not diefine the various grades of reactions, but the high percentage showing no reaction would suggest that the operation was comparatively harmless.

TABLE 1.-PERCENTAGE OF REACTIONS OBSERVED BY

\begin{tabular}{|c|c|c|}
\hline MAURANGI & & \\
\hline ypes of Rea & $\begin{array}{c}\text { Typhoid } \\
\text { Percentage }\end{array}$ & $\begin{array}{c}\text { Para-B } \\
\text { Percentage }\end{array}$ \\
\hline$\ldots \ldots$ & 92.23 & 98.59 \\
\hline & 6.18 & 1.41 \\
\hline ate & 1.40 & 0.00 \\
\hline nced & 0.19 & 0.00 \\
\hline
\end{tabular}

The general reaction is usually accompanied by fever, the most common rise being to $38 \mathrm{C}$. (100.4 F.) or slightly higher. The symptoms occur within a few hours, beginning with depression, headache, anorexia, pains in the joints, and diarrhea. The most persistent of these symptoms is headache; but malaise frequently lasts forty-eight hours, and may be accompanied by anorexia. This degree of reaction is common in from 4 to 10 per cent. of robust men.

Severe reactions are usally attended by fever of several degrees, and by one or more of the following symptoms: rigors, vomiting and diarrhea, catarrhal icterus, prostration and collapse. These reactions may 
persist for as long a period as fifteen days, and may occur after any one of the individual inoculations. The percentage of occurrence, however, is very small. To avoid these reactions it is necessary to be alert as to the contraindications to the vaccination.

Contraindications.-The age of the subject is of but little importance as a contraindication. Maurange states that he has carried out inoculations in individuals of from 4 to 46 years of age. Children show no reaction.

Contraindications may be divided into physiologic and pathologic groups. The most frequently occurring physiologic condition to be regarded as a contraindication is fatigue. It has been noted by most authors that men who are worn out from work and lack of food are prone to have a more severe reaction.

Among the pathologic conditions to be considered are angina and bronchitis, together with malaise or any of the prodromes of acute or chronic infections. The thermometer is here a valuable ally.

Patients with paludism and chronic syphilis need not be exempted from vaccination; but old tuberculous cases merit careful examination, and if the least doubt exists, owing to a history of hemoptysis or pleurisy or to the findings of anemia, rapid pulse, or fever, the patient should not be vaccinated.

Organic diseases to be enumerated as contraindications are noncompensating renal disturbances, diabetes, noncompensating myocardial and endocardial involvements, aortitis and arteritis, cachexias, gastro-intestinal disturbances, and alcoholism. Contraindicatory signs are high blood pressure, albuminuria, tender liver, and abnormal pulse findings.

A severe reaction to a previous inoculation should be considered a contraindication. There is, however, no objection to the administration of the first inoculation during the incubation period eight days immediately previous to the onset of typhoid fever.

With these contraindications in mind, the precautions to be observed in conducting the vaccination are evident, but may perhaps be emphasized with advantage.

Precautions.-Chief among these precautions is rest, for at least two days, for men vaccinated. Vincent attributes the lack of severe reactions in the Japanese experiences to the routine of two days' rest in bed following the injection, and the selection of the men vaccinated. A selection of men can be rapidly made by a query as to how they feel. Even this precaution serves to prevent the inoculation of those with malaise without fever who are in the incubation stage of acute infectious diseases, such as meningitis. Men concerning whom there are doubts should be examined carefully, including an interrogatory, observation of evening temperature, physical examination of the heart and lungs, and a urine examination. In one series, a routine urine examination of 6,040 young men revealed forty-four unsuspected albuminurias.

Goldscheider, Vincent and Maurange severally recommend, in doubtful cases, a test dose of 0.25 c.c. of the vaccine.

Maurange uses the pulse rate in repose as a criterion for the vaccination of tuberculous subjects, vaccinating those with a pulse rate of from 70 to 80 , and not vaccinating those with a pulse rate over 90 . He states that he has immunized 127 tuberculous patients without untoward results, and that persons with joint and bone tuberculosis stand the vaccination well.
The failure properly to select men for inoculation has resulted in some untoward complications following the vaccination.

Complications - The complications noted have been those of arthritis, nephritis accompanied by hematuria and uremia, and pulmonary and intestinal involvements. The most frequent pulmonary disturbance was that of a dry, diffuse bronchitis. In several cases there was a recrudescence of pulmonary tuberculosis in which the fever remained high for a long time. Chantemesse has emphasized the occurrence of the recrudescences of tuberculosis during antityphoid immunization.

The severe reactions and untoward complications attendant on the neglect of the precautions emphasized have done much to retard the general adoption of a most efficacious prophylactic measure. It should be considered that not every one can be vaccinated against typhoid fever.

Among those, however, even in the presence of contraindicatory findings, who have been carefully vaccinated, the results are most favorable in the effect on the course of the disease, and perhaps on its dissemination.

\section{COURSE OF THE DISEASE}

The effect on the course of the disease is well established. The duration of the fever is lessened. The percentage of patients showing a temperature of 39.6 C. (103.3 F.) or over is lessened by about fourteen, in a study of 300 cases. The structure of the fever curve is the same in the vaccinated as in the nonvaccinated; but the duration of the fastigium is shortened, and in the vaccinated, it is more often absent. The fever curves of the vaccinated are more frequently those of light cases. A remitting form of fever is, however, more common in the vaccinated; also a condition of typhoid infection without fever is more common. This condition is one in which the patient's fever disappears, but malaise, diarrhea and rose spots persist.

The general condition of the patient is better in the inoculated than in the uninoculated. The toxic cerebral symptoms are lessened. The heart, pulse and respiratory activity are more favorably maintained. Complications are rarer in the inoculated, especially heart muscle affections, phlebitis and enteric hemorrhages, otitis and other purulent processes. There is no lessening of the recurrences by inoculation. Convalescence in the inoculated is frequently strikingly light and rapid.

Two inoculations seem to affect these changes more favorably than one, and three more favorably than two; four inoculations seem to have no greater effect than three. It is to be noted that these modifications correspond closely with the clinical pictures of paratyphoid infections; hence, a bacteriologic diagnosis is necessary for accuracy in the determination of the effect that antityphoid vaccination may have on the course of typhoid.

According to several investigators, who have made careful studies of agglutination curves in typhoid infections, among both the nonvaccinated and vaccinated, the determination of both diagnosis and immunity is most uncertain by this serologic test. The serums of many patients never agglutinate the infecting organism, and others lose the power to agglutinate before convalescence. Intercurrent febrile conditions, such, even, as angina and rhinitis, are sufficient to increase the agglutinating titer in the serum of the 
vaccinated. Vaccination after a previous case of typhoid, even as remote as twelve years, tends to increase the agglutination titer of the serum. Hemoculture has been adopted as the safest criterion for the determination of the infection, and no test of immunity, other than exposure and reinfection, has been evolved. Positive findings by hemoculture are less frequent in the vaccinated.

The effects on the dissemination or morbidity of the disease cannot be shown with any accuracy from the reports which are at hand, and only a compilation, during times of less stress, of the experiences of the many military medical officers involved in this study will enable us to form an intelligent opinion. The reports submitted are fragmentary, usually emphasizing the effect on typhoid morbidity and mortality of one, two or three inoculations, in contrast with the nonvaccinated, without including any reference to the percentage of incidence in the entire division or corps of men, or even to the percentage of vaccinated in the nontyphoidal affections. Further, there has not been a scientific separation of the typhoid fevers, in many of these reports. However, in such careful and complete analyses as have been made, there seems to be a 60 to 70 per cent. protection against infection.

The mortality has been even more reduced, since the case mortality is about three times as great in the nonvaccinated as in the vaccinated.

English reports state that up to January, 1916, 1,365 cases of typhoid have occurred in the British army in France and Belgium. Further, up to 1916, the death rate for typhoid among the British troops in active service was less than that for the male civil population of similar age in England and Wales.

In those bodies of men concerning whom there are complete statistics with regard to the prophylactic use of the typhoid vaccines, such as in the Japanese marines and seamen, one finds a reduction in morbidity of approximately $66 \%$ per cent, and an equally great reduction in mortality.

As indicated above, final conclusions cannot be drawn from the reports at hand. One of the great disparities in these reports is the failure to indicate what is meant by typhoid fever.

The separation of typhoid fever into three distinct infections was effected a long time ago, but the prevalence of paratyphoid, and the interrelations of typhoid and paratyphoid have become manifest much more recently. And only during the war have the paratyphoid infections become of great importance.

Widal and Courmont regard paratyphoid among the troops as the most prominent epidemiologic fact of the war.

Lehman, in a review in 1916 of our knowledge of the geographic distribution of paratyphoid A infections, considers this variety of paratyphoid to have had, previous to the war, a distribution relatively limited, in comparison with typhoid. He considers it a disease of tropical and subtropical Asia and Africa, and is dubious concerning the reports from Europe and America of the presence of the organism in food, in the intestinal canal of animals, in water, and in milk. He believes that its dissemination is chiefly through the medium of "carriers," especially as it usually runs a light course, and presents many ambulant cases.

The expeditionary forces at the Dardanelles had a considerable incidence of paratyphoid infections among them.
Landouzy, in December, 1914, reported a predominance, in certain districts of France, of the paratyphoid fevers over the typhoids.

Bernard and Paraf, in an analytic study, reported in 1915 a great preponderance of paratyphoid infections in the antityphoid vaccinated over those in the nonvaccinated, presenting the remarkable figures given in Table 2. Unfortunately these authors do not give the relative proportion of the normal contingents who were given the antityphoid vaccination. Rist, however, in 1916 reported a careful analytic study of a small series of 215 cases of typhoidal infection with 621 controls of nontyphoid diseases, among which he determined the percentage of antityphoid vaccinations. $H c$ finds that the percentage of protection afforded against infection with typhoid is offset by the same percentage of increase in paratyphoids. This percentage is identical, to within a figure in the second decimal place.

TABLE 2.-PREPONDERANCE OF PARATYPHOID INFECTIONS OVER TYPHOID

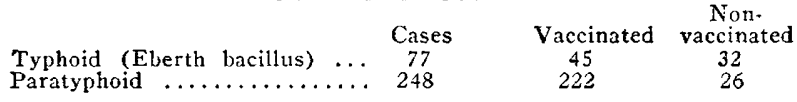

Labbé also reports in 1916 the figures concerning antityphoid vaccination given in Table 3 .

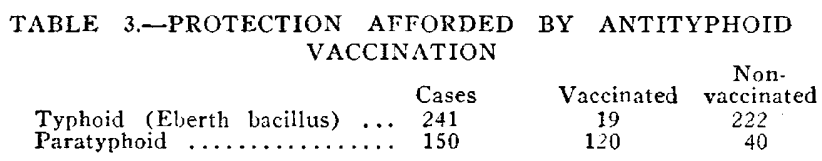

These French experiences, together with those of the expeditionary force at the Dardanelles, have shown the relative futility of vaccinating with the simple typhoid vaccine, and have resulted in the serious consideration, and to some degree, adoption by the French of a triple vaccination, simultaneously administered, or of a triple mixed vaccine.

\section{MIXED VACCINES}

Experimentation by Widal and others has shown that vaccination of animals with equal parts of the three heated vaccine viruses (typhoid, paratyphoid $A$ and paratyphoid B) produces in their blood evidences of simultaneous immunization against the three infections.

Kabeshima reduced the morbidity of typhoidal fevers among the Japanese marines by injecting separately the vaccines of typhoid, and paratyphoid $A$ and $B$. He states that the simultaneous injection of the three vaccines was not attended by a greater percentage of severe reactions in experimental animals or men than was the injection of the simple typhoid vaccine.

Independent of the vaccinations practiced with this method against the three diseases, the Japanese physicians have had recourse to another preparation, a triple vaccine against typhoid and paratyphoid $A$ and $B$ prepared by mixing in equal parts each of the three heated viruses.

From his experiments on guinea-pigs with the use of this mixed triple vaccine, Kabeshima has established the fact that these animals acquired a resistance to the virus of each of these diseases equally as strong as when the vaccinations were made with the separate vaccines. 
With respect to man, the injection of the triple mixed vaccine has been effective aboard the Japanese war vessel Katori. What the results were in these sailors, from the point of view of effectiveness, after vaccination with this mixed triple vaccine, the author does not state; he speaks only of the reactions which were caused by this triple method.

Reactions and Inconveniences of This Vaccine.This combination of the three vaccines raises another question.

The persons who receive the triple vaccine, according to the Japanese authors, do not show local or general reactions appreciably stronger than those caused by a dose of the simple vaccine. It is known, however, that these reactions last at least two days, and that the number of the vaccinated who show a fever between 39 and 40 C. (102.2 and 104 F.) after the triple vaccination is twice as large as after the simple vaccination. According to Kabeshima's figures, the proportion is about 5 per cent. of those inoculated, with the use, however, of six times the amount of bacilli per cubic centimeter as in the simple vaccinations.

Vincent, in describing previous experiences of the French with a triple mixed vaccine, and commenting on the procedure, with the view of adopting a method of triple immunization, gives a comprehensive criticism of the problems involved. He says:

In 1913, and also in 1914, it was further used at Oudjda under the direction of Dr. Grenier; the respective proportions of the three antigens was in 1913: typhoid vaccine, twothirds; paratyphoid A or B, one-third; in 1914, equal parts of these. . .

The immunity conferred on men, to the number of more than 4,000 , who have received the mixed vaccine, was shown to be as effective against paratyphoid fevers as against typhoid. The same results have been reported in the Italian army:

It has been clearly established, by observation made in man, that the protective effects due to the three mixed vaccines are not neutralized. They are superimposed in the same way when one vaccinates simultaneously against typhoid and against smallpox, a common practice in France, or against cholera and smallpox. . . .

It may be stated here, that if a really effective immunizing result is to be obtained with the mixed vaccine, it is self-evident that it is necessary to inject simultaneously the necessary dose of each vaccine in order to produce the immunity against each of the three diseases.

It follows, then, that if one seeks to obtain at once the triple immunity, the advantage can be counterbalanced by a practical inconvenience, not at all to be ignored, namely, the increase of the number of local and general reactions.

Such is in fact the factor which may hinder the generalization of the immunity with a mixed vaccine.

Dr. Grenier has stated to me that in the last attempts which were carried out at oriental Morocco in 1913 and 1914 , the reactions in the soldiers vaccinated with the mixed vaccine were moderate, hardly more severe than those caused by antityphoid vaccine alone; but for vaccination a selection of the men had to be made, those who were well rested.

Under the present conditions, in soldiers recently enlisted exposed to the fatigue of military initiation, marches, daily drills, night marches, or those stationed in the trenches or in the neighboring camps and exposed to the conditions of war, it appears wiser to make series of the antityphoid vaccinations and the antiparatyphoid, except under special conditions which permit the mixed vaccination.

This practice of successive vaccinations has been carried out a large number of times, notably at Val-de-Grâce. Those who have been successively injected with the different vaccines have not complained of fatigue and have not shown abnormal febrile reaction. They have appeared regularly for the full series of injections.

Moreover, it is possible greatly to simplify this practice. A good immunity can be obtained with two injections of antityphoid vaccine. This reduced vaccination, made with doses a little increased, was practiced on my recommendation on a large number of men at the beginning of the war. It has been very effective. These two antityphoid injections can be then followed by two or three injections of antiparatyphoid vaccine $A$ or $B$, or $A$ plus $B$. This last mixture, which contains only two vaccines is well supported.

We endeavor, for this reason, to extend the employment of the antiparatyphoid vaccine when it is necessary. In special cases, of which the vaccinating surgeon is the best judge, the mixed vaccine can be used. In a general way, it appears preferable, especially under the conditions stated, not to extend this practice systematically; especially when it is possible to make successive vaccinations.

Widal and Chantemesse concur in this opinion on the use of a triple mixed vaccine. (Incidentally the method of separate triple vaccination is that practiced in the American army.)

The paratyphoid fevers are common in the Italian army, and pursuant to the advice of Vincent this army has been using his etherized, mixed, triple vaccine since 1912.

Tarrasevitch states that the Russian army made use of simultaneous vaccination against the three typhoids in the later vaccinations; and further, that in Moscow, 25,000 men received simultaneous vaccination against the typhoids, cholera and smallpox with only 5 per cent. presenting a moderate or severe reaction.

Castellani states that 170,000 Serbian soldiers were vaccinated with a combined tetravaccine recommended by him, which included cholera together with the three typhoids. The vaccines were prepared by killing twenty-four hour agar cultures suspended in salt solution with phenol.

Reports on the use of anticholera vaccination are confined largely to experiences on the eastern front.

The anticholera antigens are prepared by heating suspensions of twenty-four hour agar cultures in physiologic sodium chlorid solution and by phenolizing these; or only by phenolizing the suspensions, adding phenol to a strength of 0.5 per cent. The official German vaccine contains two oese of culture in a cubic centimeter. Killed organisms are always used, as opposed to Haffkine's method of vaccination with attenuated live organisms; strains are chosen from the local epidemics.

The vaccine contains usually $2 \mathrm{mg}$., wet weight, of organisms to the cubic centimeter.

The dose administered is 1 c.c., followed in from five to seven days with a second dose of 2 c.c.

Anticholera vaccination has been made compulsory in the Austrian army, and provisions have been made for vaccinating the civil communities in the area of military operations. Reinoculation for cholera has been instituted after three to four months, since the immunity is evanescent, apparently lasting only from six to seven months.

Bujwid, who used a sensitized typhoid vaccine and phenolized cholera vaccine, notes that the typhoid reaction is more severe than the cholera reaction, the latter producing a painful swelling in a few hours, with a rise in temperature to $38 \mathrm{C}$. $(100.4 \mathrm{~F}$.), rarely to $39 \mathrm{C} .(102.2 \mathrm{~F}$.$) , and is of shorter duration than$ that of the typhoid. 
Bujwid states that anticholera vaccination is not as efficient as antityphoid vaccination. Hoffman, however, reported that among German troops, when advancing in the regions of the Bug and Rokitano marshes, the mortality among the uninoculated was from 35 to 50 per cent., while among those inoculated it was from 0 to 20 per cent.

Kaup (Austrian) stated that after the soldiers had been inoculated, the morbidity was from 1 to 5 per cent., though the exposure was great. The course of the disease was mild, and the mortality was from 0 to 20 per cent., as opposed to from 40 to 60 per cent. in the uninoculated.

A Polish newspaper, in commenting on a winter epidemic of cholera in Poland, remarks, "Cholera seems to be a disease which attacks the civil population and spares soldiers."

While anticholera vaccination has been restricted largely to its use among troops operating on one front, the results obtained are favorably comparable to those obtained from antityphoid vaccines.

Concerning the third important intestinal disease, dysentery, reports are very sparse.

\section{DYSENTERY}

Antidysentery vaccinations have apparently not been widely practiced.

Hever and Luksch report their use in a small epidemic in a civil community in Germany. The strains found were both the Shiga-Kruse and the Flexner. Their results are not convincing.

The English have been experimenting with several chemicals for the detoxication of the Shiga-Kruse types, and have some preparations of vaccines made in this manner.

Castellani recommends a polyvalent vaccine containing the strains of Shiga-Kruse, Flexner and Hiss-"y," to be prepared by killing twenty-four agar cultures suspended in salt solution with 0.5 per cent. phenol, and administering doses of 125 million organisms, contained in 0.5 c.c. of suspension.

Hever and Luksch found that children bore the antidysenteric vaccination well with slight or no symptoms, and that no untoward effects were produced in adults.

Landouzy warns us that much of the "trench diarthea and dysentery" is caused by paratyphoid infection, in which diarrhea is practically a constant finding.

\section{CARRIERS}

The modification of the usual course of these diseases, to that of a mild febrile disturbance, with many ambulant cases, or to one without symptoms, recognized only by a routine bacteriologic diagnosis, has introduced another problem for the military and for civil communities - the "carrier" problem. Many of these vaccinated patients become carriers, especially those vaccinated against cholera.

Kaup, who had a large experience with cholera, warns of the danger of the carrier, especially with regard to his transportation to prison camps and to civil communities. He states that many carriers develop in new troops vaccinated, and coming to the front in the presence of cholera.

Rosenthal and Werz, who made some studies in the German army, found nearly 2 per cent. of carriers in a general examination of 686 wounded. In 248 cases examined, the cholera vibrio was found in 3 per cent. of those inoculated twice. Of the carriers, nine showed no agglutinins in their blood, and but two a weak reaction. They found difficulty in distinguishing between the carriers and the light cases.

\section{SUMMARY}

The present war has emphasized the inadequacy of general sanitary measures, without individual imnnunization, in the prevention of the infectious diseases of greatest military importance, namely, the intestinal infections.

The problems in this immunization are those common to all artificial immunization, modified by the readiness with which the hosts lend themselves to the reaction to the group of organisms involved:

The antigens used during the war have varied somewhat in respect to preparation and dosage. However, they have had as the basic principle for their preparation the choice of that method which conserves the immunogenic characteristics, with a destruction of their infectivity.

There seems to be a tendency toward increasing the dosage of the antigenic principles, based on the facility of administration of the entire dosage, at one inoculation, and on the belief that the mass of vaccine injected is important.

The period of immunity, after these vaccinations, is in general shorter than has been supposed hitherto, as determined by Wright's observations in India.

The methods of vaccination practiced are both enteral and parenteral. The former method is of limited usage, and does not at present promise good results.

The parenteral injections are made subcutaneously, and can be carried out very rapidly. With the proper selection of robust men, preferably under 40 years of age, this method is unattended by severe results.

The results of the vaccination may be variously considered from the standpoint of the effects on the subject, on the course of the disease in the host, and on the frequency with which the disease attacks, and on its rapidity of dissemination.

The subject reacts locally by redness and swelling, and, in a small percentage of patients vaccinated, by a general reaction.

The general reaction may be light, consisting of headache, anorexia, and slight fever; or severe, with a mild reproduction of the typhoid fever syndrome, or with more toxic symptoms.

These severe reactions, as well as complications attending them, can be largely avoided by attention to contraindicatory signs and conditions.

Such contraindications are both physiologic and pathologic. Chief among the physiologic conditions is fatigue; and among the pathologic states are those conditions prodromal to acute and chronic infections, together with organic, respiratory, renal or circulatory involvements, and systemic diseases.

Failure to adopt the precautions of rest following vaccination, and of a selection and examination of men about whom there is doubt concerning their fitness for vaccination, may lead to such complications as nenhritis, pulmonary and intestinal involvements, and even the recrudescence of a subsiding or latent tuberculosis.

The effect on the disease is to shorten its duration and to mollify its intensity. While such positive statements cannot be made with regard to the protection afforded against infection, there is sufficient evidence 
to show that both the morbidity and the mortality are greatly decreased.

The diagnosis of typhoid fever has been made more difficult by vaccination, since the serologic reactions usually found are altered by vaccination. Hemoculture affords the best criterion for diagnosis, and here again vaccination has interfered by making the cultivation of the causative organism from the blood apparently more difficult.

Careful studies have shown that, with the separation of the typhoid fevers, there has been a tremendous prevalence of paratyphoid infections current, with reduction of infection by the bacillus of Eberth following vaccination. Since no protection seems to be afforded by simple antityphoid vaccination against the paratyphoid infections, triple immunization is recommended and practiced by simultaneous injection of vaccines of each of the organisms, or by injection of a mixed vaccine containing all three.

The method of triple immunization has been found to be efficient in reducing the morbidity and mortality from these fevers in the Japanese experience, and in that of the French at Morocco. The reactions attending this method are not of much greater severity than those of the simple antityphoid vaccination.

Anticholera vaccination has been limited to the troops engaged on the eastern front. The vaccines have been made of killed cholera vibrios, of local strains. They are administered in two doses, and are productive of less severe reactions than the typhoid vaccinations used on the Austrian eastern front.

The immunity is more evanescent, necessitating reinoculation after an interval of from three to four months.

The results of the vaccination were favorable on the course of the disease, its morbidity and its mortality.

Antidysentery vaccination has been but slightly practiced.

The modification of the course of these intestinal infections, to that of a mild febrile disturbance, has produced many "carriers" and has thereby created an additional serions problem for both the military and civil communities.

\section{ABSTRACT OF DISCUSSION}

Dr. Fred I. Lackenbach, San Francisco: Will Dr. Wayson explain the matter of dosage? If, in using mixed vaccines, he doubles or trebles on the dosage over the single strain? Also, if by "mixed" vaccines he refers to the Bacillus paratyphosus A and B, as well as the Bacillus typhosus? Also, has the United States government adopted the use of the mixed vaccines in its practice?

Dr. J. F. Anderson, New Brunswick, N. J.: I should like to ask Dr. Wayson if the Besredka vaccine was a living vaccine or a killed vaccine, and the periods of potency of the vaccine used.

Dr. Herman Spitz, Nashville, Tenn.: Regarding Dr. Wayson's statement that the use of a vital reaction is unreliable in these conditions, I should like to report that in a series of some 200 patients (including both sexes and all ages) who received the prophylactic vaccine, the Widal reaction was uniformly negative. The vaccine used consisted of two strains of B. typhosus and one each of B.paratyphosus A and B. Three and four doses were given. I want to ask Dr. Wayson if he can offer some explanation why the Widal is negative.

Dr. Walton F. Dutron, Tulsa, Okla.: For the benefit of those who practice in malarial districts I would cite one case. Last summer I treated a patient with estivo-autumnal fever with quinin. The patient recovered from the attack of malaria, but did not appear to improve in general health. The condition simulated a pretyphoid state. Typhoid prophylactic vaccines were used and the patient began to improve rapidly and made a good recovery.

Dr. Carl C. Warden, Ann Arbor, Mich.: I believe that ultimately the solution of the healing of infectious diseases will be attained through these vaccine therapies; and I believe the most recent advances are those outlined by Dr. Miller today. The question how vaccines work comes up. Recent studies on the toxicity of human and other serums have shown the following facts: that blood lymph or whole blood or serum or plasma may be rendered toxic by almost any substance brought in contact with it. In other words, the serum may be rendered toxic not only by bacteria, but also by certain protein solutions, by substances such as agar, starch, charcoal, kaolin or any other substance which possesses surface. Studies show that a serum so rendered toxic will produce certain symptoms in some animals but not in other animals into which the serum is injected, or will reproduce the same reactions in the body of the animal as in the diseased body. These are anaphylactic reactions and may be produced in any degree. This seems to indicate that the action of the substance is a physical one, as the same reaction may be produced by any substance. Two serums may differ as to accomplishing the same thing because of variation in the precipitation of the globulins. The injection of vaccines into the human being apparently excites a definite reaction which must be regarded as anaphylactic shock, and this anaphylaxis is part of the immune reaction going on in the body as a result of infectious disease; but that is not all of the immune reaction; accordingly, such treatment as is applied to infectious diseases will yield a certain percentage of good results in a large number of cases, but will not cure all cases of infectious diseases. Granting that these reactions are part of the grand immunity process, we have evidence to show that a great many so-called immune reactions are also part of the same grand immune reaction. Precipitins and agglutinins, complement fixation and opsonins and anaphylaxis may be regarded as representing different phases only of the same large phenomenon of immunity. If we consider such reactions as precipitins or complement fixation, we can show that they occur between three substances-the antibody, the complement, and the antigen which permits a definite reaction to occur with the adsorption of complement. In syphilis and gonorrhea, to which I particularly refer, we have such immune reaction depending on the specificity of an appropriate antigenic substance of a fatty nature. When experimentation on infections shall have progressed further, then the application of vaccine therapy to infectious diseases will start on its right course.

Dr. Newton Wayson, Washington, D. C.: The dosage of mixed vaccines varies considerably in practice. There is, however, the usage of the same proportion of each organism, simultaneously given, in a maximum of 500 millions in the first dosage, 1,000 millions in the second. There is a tendency to make the dosage larger, rather than smaller. The United States practice I cannot give you authoritatively, but I am informed that all troops will be vaccinated against typhoid and paratyphoid, and the mixture of paratyphoid $\mathrm{A}$ and $\mathrm{B}$ and typhoid will represent one third and two thirds, respectively. It will be a simultaneous triple vaccine and not a simple mixed vaccine. The simple typhoid vaccine will be given in the usual dosage. By mixed vaccine $I$ mean a vaccine composed of typhoid and paratyphoid $A$ and $B$. By triple simultaneous vaccine, I mean vaccines by three injections: one of the typhoid, one of the paratyphoid $\mathrm{A}$, and one of the paratyphoid B. The Besredka vaccine is not a killed vaccine. The period of potency of vaccine is uncertain. Weber and Wassermann, who have done considerable experimental work, think a vaccine should age three weeks, and that beyond this, the age may be almost indefinite without affecting potency. Their experimental work was on laboratory animals.

The unreliability of the Widal reaction is dependent on two factors. I cannot explain why the agglutinins disappear; but it has been found on careful analyses in typhoid fever that the Widal reaction occurs at very irregular periods, 
appearing as late as the eighteenth or twentieth day of the disease, as early as the seventh or eighth day, and sometimes never occurs. Also, first to occur may be a predominant agglutination for the typhoid bacillus, later a predominance for the paratyphoid $A$ or $B$, and later a rising agglutination for the paratyphoid A or B bacillus. A second factor which makes the diagnosis unreliable is that a patient vaccinated against typhoid may show toward the typhoid bacillus a high agglutination titer, which disappears, to return when the patient is affected with a slight bronchitis.

\section{WAX PARAFFIN FILM IN THE TREAT- MENT OF BURNS*}

M. L. EMERSON, M.D.

Chief of Surgical Staff, Samuel Merritt Hospital

OAKLAND, CALIF.

Following the suggestion of Dr. D'Arcy Power, and using his formulas for a film, I have completed treatment in 100 out of about 250 cases treated. After the hundredth case I ceased to record the histories. The treatment was used in certain selected cases in which it seemed most indicated. The patients were selected from my service at Merritt Hospital and the Union Iron Works. Brush burns or superficial abrasions or epithelial scraping of skin surfaces are included in this number, burns from electric current, fuse explosions, hot rivets, molten metal, oil flames, hot bitumen, steam, scalds and dry heat, as well as granulating surfaces. Burns are usually localized, while the scalds are more extensive. Their pathology being the same, the treatment will be considered together. For the sake of brevity, these various burns will be considered under three headings: first degree, those which involve the superficial layer of the skin characterized by reslness without vesication; second degree, those which involve the corium and are characterized by the formation of vesicles and bullae, and third degree, those which destroy the entire skin and partially or completely carbonize fat, fascia, muscles and underlying structure.

\section{FIRST DEGREE BURNS}

Case 1.-J. R., rivet heater, had a flash burn on the side of the neck from a sudden explosion of crude oil. The burn extended from the maxilla down to the shirt collar from the front of the neck to well back under the ear. The first treatment consisted of cleansing the part of grease and dust particles with 0.5 per cent. iodin in benzin. The part was then thoroughly dried with a small warm air electric blower, such as hair dressers use for drying the hair. A very fine filament of wax was now sprayed over the surface. No other dressing was applied. The application relieved the pain. After three similar applications at intervals of twentyfour hours, the patient was discharged as cured. The film in this type of case must be very thin. A thicker coating without a gauze dressing will curl up and peel off.

CASE 2.-A similar burn covering the entire back of the ncck was treated in the same way, and the patient was discharged after two applications.

CASE 3.-A flash burn the size of a dollar on the left check was given three applications, after which the patient was discharged.

There being no broken surface and no chance for infection, the type of burn illustrated in Case 2 generally is cured in a few days with any of the recognized treatments. Carbonized carron oil, phenolated grease or petrolatum, starch and soda, anything that

* Read before the Alameda Medical Association. excludes the air, generally gives relief. These burns are seldom covered by dressings unless they are on the hands.

\section{SECOND DEGREE BURNS}

These are the most common, over 70 per cent. being tabulated out of the entire group. The majority are infected, especially if oils and medicated grease are applied as a first dressing. Carron oil, composed of equal parts of lime water and sweet oil, is popular among mechanics, but it is the bugbear of the surgeon. Many patients treat themselves first, and then come to the clinic for treatment of the infection.

CASE 4.-A second degree burn on the anterior surface of the leg, 3 by 4 inches square, was treated for seven days by moist dressing. The skin was thoroughly dried, every pocket of stiperficial skin was removed with cuticle scissors, and the wax film was applied. A considerable discharge looking like yellowish pus followed the first dressing, but this soon cleared up.

The continued moisture collecting under the wax seems to be one of the great disadvantages. However, this wound healed kindly under twenty-seven days' treatment of daily wax application, which I consider a fair result. It would probably have healed just as quickly under a moist dressing of physiologic sodium chlorid solution, sodium bicarbonate solution or a 1 per cent. watery solution of picric acid, the advantages of the latter being that the dressings can often go from two to five days without being changed.

CASE 5 .-A brush wound covering the entire outer aspect of the right arm from the shoulder to the elbow, superficial in character yet one which should be classified as a second rlegree injury, showed but little contusion; the superficial abrasion was caused by the breaking of an air hose, causing a friction rub over the outer aspect of the arm denuding it of the superficial epithelium. The wound healed completely under six daily applications of wax.

This I consider an ideal case for wax treatment. This type of wound is not so serious as it looks, especially if the deeper structures of the skin have not been tratumatized, and there is no extensive hemorrhage from the surface. They always become infected if grease is applied, and it is always tempting to place a miedicated salve over this type of injury. It requires two or three weeks to heal up the infection, especially if the grease application is continued. These wounds do well under a dry application of dusting powder, one part of salicylic acid to three parts of boric acid. However, the wax worked admirably in this case, probably better than any other form of treatment would have done.

CASE 6.-A foot burn covering the anterior aspect of the foot from the ankle to the toes, while classed as a second degree burn, presented three or four small areas that were much deeper which seemed to be very slow in healing. Many of these burns could be classified as first, second and third degree. In these small areas I placed small pledgets of gauze saturated with phenolated camphor, and then covered the entire area with wax. This worked nicely. The burn healed under fourteen daily treatments of the wax film.

CASE 7.-A burn on the back of right hand, the size of a silver dollar, seemed very stubborn under the wax treatment, requiring thirty-seven daily treatments. I imagine it would have healed much faster under a picric acid or boric acid compress.

The great majority of all patients with burns continue working. One can well imagine how slow the healing must be in a malleolus burn the size of a silver 